\title{
Implicaciones medioambientales y socioeconómicas de las energías renovables
}

\author{
Carlos J. Pardo Abad *
}

\begin{abstract}
RESUMEN
ABSTRACT

El impacto de la energía sobre el medio ambiente es profundo y cada vez más complicado de resolver. Los problemas afectan a distintas escalas geográficas y demandan la participación más activa de las energías renovables. Los beneficios no sólo son medioambientales sino también económicos y sociales. reducción de emisiones contaminantes a la atmósfera, creación de empleo, impulso tecnológico, incremento de la

competitividad industrial, modernización y desarrollo de recursos propios en áreas rurales, etc.

PALABRAS CLAVE: Energía, recursos renovables, medio ambiente y empleo.

The impact of energy on environment is deep and it is becoming more and more complicated to resolve. The problems affect several geographical scales and require a more active participation of renewable energies. Benefits are not only environmental, but also economic and social: reduction of pollutant emissions to atmosphere, creation of new jobs, technological boost, increase of the industrial competitiveness, modernization and development of own resources in country areas, etc.

KEY WORDS:

Energy, renewable resources, environment and employment.
\end{abstract}

* Departamento de Geografia de la UNED. 


\section{LOS IMPACTOS DEL ACTUAL SISTEMA ENERGÉTICO}

Las actividades humanas, cada vez más intensas en los países desarrollados, demandan crecientes volúmenes de energía y ocasionan graves problemas de contaminación del medio ambiente. Este consumo, a pesar de los avances conseguidos, sigue aún muy centrado en las energías no renovables, es decir, los combustibles fósiles. Este sistema energético es muy intensivo: por un lado, por el volumen de consumo alcanzado y, por otro, por las enormes cantidades de contaminantes emitidos a la atmósfera. Las consecuencias ambientales son evidentes y se pueden anotar algunas agresiones de gran trascendencia, como el aumento general de las temperaturas y las precipitaciones ácidas.

Los impactos se han incrementado a la par que se ha intensificado la demanda energética, desmedida en muchos casos y con niveles de consumo que bien cabe definirlos como auténtico derroche. Los programas de eficiencia y ahorro energético (que han proliferado en todos los países desarrollados) no han sido suficientes para frenar el consumo y en ello ha jugado un papel muy importante, no hay que olvidarlo, la existencia de unos precios relativamente baratos para el conjunto de la energía y la falta de un auténtico compromiso a nivel internacional.

Las repercusiones de la energía sobre el medio ambiente son, si cabe, más numerosas que las propias fuentes energéticas utilizadas por la sociedad (Lucena, A., 1998). Las soluciones son cada día más complicadas y sus efectos cada vez más amplios, afectando a distintas escalas geográficas. Algunos autores señalan tres niveles diferentes según los problemas presentados de tipo energético (Menéndez, E., 1997): de ámbito local, de ámbito regional y los de ámbito global.

Los locales (la contaminación de las concentraciones urbanas e industriales, por ejemplo) se dan en entornos geográficos reducidos. El uso de combustibles ocasiona la expulsión de óxidos de azufre y de nitrógeno, partículas, metales pesados, monóxido de carbono, etc. Como el número es elevado se pueden producir incluso combinaciones de elementos distintos, ampliando las posibilidades de incidencia sobre el medio. Un ejemplo es la reacción de los óxidos de nitrógeno y los hidrocarburos con la formación de ozono en las capas bajas de la atmósfera activada por la luz solar. La alta capacidad oxidante del ozono lo convierte en un agente muy nocivo. Esto es importante en las ciudades de latitudes bajas, en donde la radiación solar es muy elevada.

El carbón y los derivados del petróleo son los que más contaminan la atmósfera urbana, siendo los efectos más reducidos en el caso del gas na- 
tural. Las instalaciones industriales también son focos contaminantes, sobre todo en el caso de la industria pesada, en donde se añaden a la combustión los contaminantes propios del proceso productivo: cementeras, plantas petroquímicas, siderúrgicas, fábricas de fertilizantes, etc.

A nivel regional (los escapes radiactivos de centrales nucleares, las lluvias ácidas o los accidentes de los petroleros en alta mar o cerca de la costa, como el reciente del Prestige en las proximidades de Galicia), la incidencia es más amplia que a escala local y puede afectar, incluso, a varios paises. Un ejemplo de este impacto es la construcción de grandes embalses: movilizan grandes masas de población, modifican la producción local de alimentos, alteran la vida acuática de los ríos, etc. En general, aunque las grandes presas (como la de Assuán en Egipto) pueden ser un motor de desarrollo económico, levantan más resistencias que las centrales minihidráulicas, que incluyen aprovechamientos de menos de 10 MW y diques inferiores a los 15 metros de altura.

Los problemas ambientales de origen energético y de ámbito global se caracterizan por afectar a grandes espacios con efectos de larga duración. El cambio climático es el principal de estos problemas y está íntimamente relacionado con el consumo de las energías no renovables. De forma natural, existe en la atmósfera una serie de gases que permiten la entrada de la radiación solar pero dificultan el escape de la radiación térmica terrestre hacia el exterior. Es un efecto invernadero natural y necesario para evitar los excesivos contrastes entre las temperaturas diurnas y las nocturnas. Los gases más significativos que intervienen en este caso son el dióxido de carbono y el vapor de agua. Cuando su concentración aumenta, se agudiza el efecto invernadero más allá de los límites normales marcados por la propia naturaleza.

La solución a estos problemas pasa por la adopción de una serie de medidas dirigidas a encuadrar cualquier toma de decisión en materia de energía y medio ambiente: mejora del rendimiento energético, utilización de técnicas anticontaminantes, sustitución de determinados combustibles y explotación y uso limpio de la energía (Pardo, C. J., 1993). Sólo de esta forma comenzará una etapa de solución a los graves problemas energéticos que tiene planteados la sociedad actual.

\section{EMISIONES CONTAMINANTES, PROBLEMAS AMBIENTALES E INTENTOS DE SOLUCIÓN}

Como ya se ha comentado, los problemas que ocasiona el consumo masivo e indiscriminado de energía son muy numerosos. Afectan 
sobre todo a los países más desarrollados, a pesar (y esto es lo paradójico) de las posibilidades técnicas y económicas existentes para la búsqueda de soluciones duraderas y eficaces. Su intensidad es tal que acaba convirtiéndose en un problema global de enormes consecuencias.

La lluvia ácida afecta a países próximos en ámbitos regionales amplios. Se empezó a tener conciencia de sus efectos en la década de 1970 en el Norte de Europa y sus daños se dejan notar en bosques y lagos. La lluvia ácida se genera a partir de las emisiones de óxidos de azufre y de nitrógeno procedentes de los combustibles fósiles; tras un proceso de oxidación, se convierten en ácido sulfúrico y ácido nítrico, respectivamente, los cuales forman las precipitaciones ácidas al combinarse con las gotas de lluvia.

La lluvia ácida afecta a extensas zonas, incluso a miles de kilómetros del foco de emisión, alterando ríos, suelos, lagos y animales acuáticos al aumentar la acidez de las aguas, y también a los bosques debido a la mayor acidez del suelo y la acción directa de la lluvia ácida sobre ramas y hojas. Los árboles ven reducida su capacidad de realizar la fotosíntesis, frenan su crecimiento y son más sensibles a enfermedades y parásitos. Las coniferas del Norte del continente europeo, desarrolladas en gran medida sobre terrenos ácidos y en zonas de abundantes precipitaciones, se han visto especialmente afectadas.

Tabla 1. Emisiones de óxidos de azufre y de nitrógeno en algunos países desarrollados. 1995

\begin{tabular}{lrrrr}
\hline \multirow{2}{*}{ País } & \multicolumn{2}{c}{ Miles de toneladas } & \multicolumn{2}{c}{ kgr. per capita } \\
\cline { 2 - 5 } & \multicolumn{1}{c}{$S O_{x}$} & NO & \multicolumn{1}{c}{$S O_{x}$} & NOx \\
\hline Alemania & 2.995 & 2.210 & 36,8 & 27,1 \\
Canadá & 2.668 & 1.995 & 91,2 & 68,2 \\
España & 2.062 & 1.223 & 52,8 & 31,3 \\
EE.UU. & 16.619 & 19.758 & 63,1 & 75,1 \\
Francia & 1.010 & 1.494 & 17,4 & 25,8 \\
Italia & 1.424 & 2.117 & 25,0 & 37,2 \\
Japón & 876 & 1.476 & 7,1 & 11,9 \\
Reino Unido & 2.360 & 2.293 & 40,3 & 39,1 \\
Suecia & 94 & 362 & 10,6 & 40,9 \\
\hline
\end{tabular}

Fuente: OCDE, Environmental Data, 1997.

Los óxidos de azufre proceden en su mayoría de las centrales térmicas de carbón para la generación de electricidad; en menor medida, de 
algunos procesos industriales como la siderurgia y la fabricación de fertilizantes. Los óxidos de nitrógeno se generan también en las centrales térmicas de generación eléctrica, sobre todo las de carbón, con la circulación de vehículos y con el uso de combustibles en la industria y usos domésticos. La solución pasa por reducir el uso de los combustibles fósiles en la producción de electricidad y aumentar de forma significativa la aportación de las energías renovables, cuyas emisiones a la atmósfera son nulas. Sólo son inevitables las emisiones de óxidos de azufre y de nitrógeno con la biomasa, pero en cantidades muy poco significativas en comparación con el carbón y los derivados del petróleo. Pero como se tiene asumido que el carbón es imprescindible en la generación de electricidad, se han investigado en profundidad nuevas tecnologías de uso limpio del carbón. Un ejemplo es la combustión en lecho fluido y la gasificación.

La combustión en lecho fluido, desarrollada a una temperatura baja $\left(850^{\circ} \mathrm{C}\right.$ para facilitar una emisión de óxidos de nitrógeno también baja, inferior a 200 ppm), deja el azufre del carbón en las cenizas de la combustión mediante el uso de un absorbente introducido en el lecho. De esta manera, se consigue reducir las emisiones de azufre en más de un $90 \%$. Mediante la gasificación, se transforma el azufre del carbón en un compuesto gaseoso de fácil eliminación y con muy bajo contenido en azufre. Además, en la combustión de este gas los niveles de formación de óxidos de nitrógeno son muy bajos. Con esta técnica se reducen las emisiones de $\mathrm{SO}_{2}$ entre un 90 y un $99 \%$.

El aumento de $\mathrm{CO}_{2}$ de origen antrópico crea una capa contaminante en la atmósfera que permite la entrada de calor procedente de la radiación solar y frena su pérdida hacia las capas altas, elevándose las temperaturas. Aunque existe mucha polémica científica al respecto, ya que no hay consenso sobre la incidencia climática del aumento de los gases de efecto invernadero ocasionado por las actividades humanas, algunos expertos han llegado a calcular un incremento térmico de 0,3 a $0,6^{\circ} \mathrm{C}$ en los últimos cien años y esperan un aumento de entre 0,2 y $0,5^{\circ} \mathrm{C}$ por decenio hasta el 2100 . Además de la modificación térmica, se han tratado otras posibles consecuencias que podrían derivarse del efecto: deshielo de nieves perpetuas, etapas acusadas de sequías y fuertes lluvias, alteración en el régimen de los vientos, cambios en la agricultura de los países más pobres, etc. Las consecuencias son tan variadas que, pese a la falta de acuerdo al respecto, hoy en día ya se habla más de cambio climático que de efecto invernadero. (Fig. 1). 


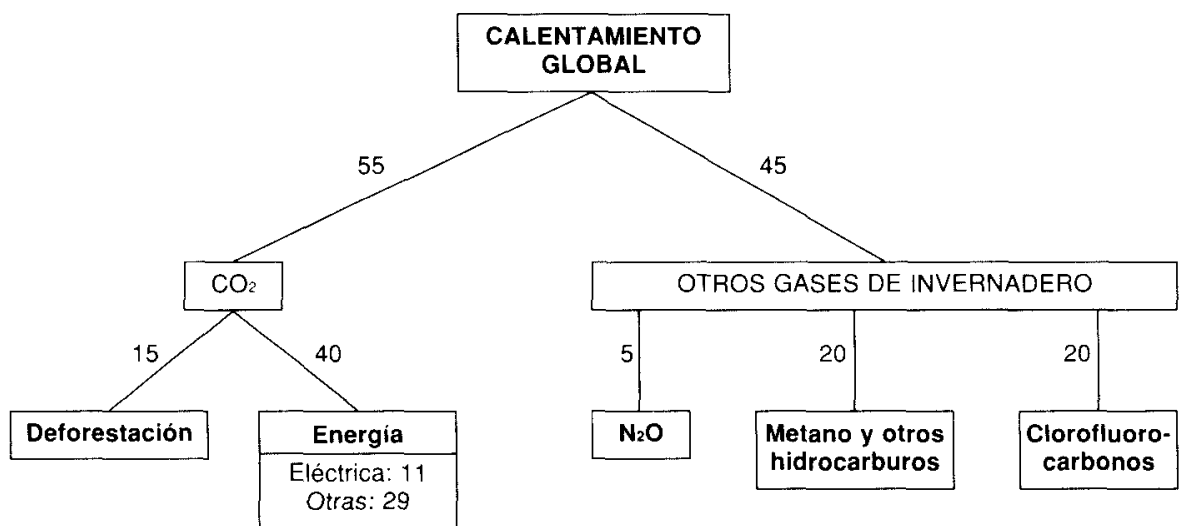

Fig. 1. Participación de las distintas fuentes y gases en el efecto invernadero (en porcentajes). Fuente: Atomic Energy Agency.

Tabla 2. Emisiones de gases de efecto invernadero en algunos paises desarrollados. 1995

\begin{tabular}{lrrrr}
\hline \multirow{2}{*}{ País } & \multicolumn{5}{c}{ Miles de toneladas } \\
\cline { 2 - 5 } & $\mathrm{CH}_{4}$ & CFCs & $\mathrm{N}_{2} \mathrm{O}$ & \multicolumn{1}{c}{$\mathrm{CO}_{2}$} \\
\hline Alemania & 4.849 & 2 & 219 & 909.410 \\
Canadá & 3.522 & 5 & 108 & 477.943 \\
España & 2.310 & 7 & 94 & 262.590 \\
EE.UU. & 28.116 & 60 & 469 & 5.263 .463 \\
Francia & 2.744 & 5 & 174 & 368.622 \\
Italia & 4.004 & 17 & 116 & 451.536 \\
Japón & 1.312 & 23 & 57 & 1.206 .938 \\
Reino Unido & 3.817 & 17 & 95 & 574.014 \\
Suecia & 245 & 0,2 & 9 & 60.606 \\
\hline
\end{tabular}

Fuente: OCDE, Environmental Data, 1997.

Tabla 3. Parámetros de gases de efecto invernadero

\begin{tabular}{lcccc}
\hline \multicolumn{1}{c}{ Parámetro } & $\mathrm{CO}_{2}$ & $\mathrm{CFCs}$ & $\mathrm{CH}_{4}$ & $\mathrm{~N}_{2} \mathrm{O}$ \\
\hline Contribución al efecto & $+50 \%$ & $20 \%$ & $12-14 \%$ & $6-7 \%$ \\
Tiempo de permanencia en atmósfera & $50-200$ años & $75-100$ años & $7-10$ años & 150 años \\
Crecimiento anual & $0,5 \%$ & $4-5 \%$ & $1 \%$ & $0,4 \%$ \\
\hline
\end{tabular}

Fuente: Foro Nuclear, 1999. 
El uso de los combustibles fósiles ha aumentado la concentración de $\mathrm{CO}_{2}$ en la atmósfera, variando en el último siglo de $250 \mathrm{ppm}$ a más de $350 \mathrm{ppm}$. Un tercio de las emisiones de este gas procede de la combustión del carbón (usado sobre todo para la generación eléctrica), otro tercio procede de los vehículos y el último se origina en la utilización de los otros combustibles fósiles y en la deforestación.

Las soluciones pasan por reducir las emisiones de dióxido de carbono, usar más racionalmente la energía, alejar el actual modelo energético del derroche y despilfarro en las sociedades más desarrolladas, incrementar la generación eléctrica con energía nuclear (aunque la oposición social al respecto sigue siendo alta) y con renovables. Sin embargo, el hecho de que el carbón sea el combustible más abundante y que muchos países subdesarrollados sólo cuenten con carbón como recurso propio indica que en un futuro a corto plazo no se asistirá a un cambio radical. Hace falta, además, asumir con valentía compromisos de reducción de emisiones de $\mathrm{CO}_{2}$ que supongan llevar a la práctica la salvaguarda de los valores ambientales contenidos en numerosas conferencias y declaraciones de ámbito internacional.

La primera reunión política sobre cambio climático tuvo lugar en Ginebra en el año 1979 a iniciativa de la Organización Meteorológica Mundial. Al amparo de esta organización y de las Naciones Unidas, se crea en 1988 el Grupo Intergubernamental de Expertos sobre el Cambio Climático (IPCC), cuyos informes de 1990 y 1995 sirvieron de base para la celebración de la Cumbre de la Tierra en Río de Janeiro en 1992 y las Conferencias de Berlín en 1995 y de Kioto en 1997.

Apoyándose en las previsiones de aumento de temperatura media y en las recomendaciones de ahorro de emisiones contaminantes del segundo informe del IPCC, la Conferencia de Berlín obligaba a las partes al compromiso de reducción de emisiones más allá del año 2000 que quedara recogida en un documento jurídicamente vinculante.

El cumplimiento de este mandato dio como resultado en 1997 el Protocolo de Kioto, en donde se establece en algo más de un $5 \%$ la reducción global de las emisiones de los seis principales gases de efecto invernadero entre el 2008 y el 2012. Estos gases son el $\mathrm{CO}_{2}, \mathrm{~N}_{2} \mathrm{O}, \mathrm{HFC}, \mathrm{PFC}, \mathrm{CH}_{4}$ y $\mathrm{SF}_{6}$. Los compromisos por países se establecen en función de dos parámetros: el mantenimiento de un crecimiento económico sostenido y la disponibilidad de tecnologías. La reducción para la Unión Europea queda establecida en el $8 \%$, para EE.UU. en el $7 \%$ y para Japón en el $6 \%$ respecto a las emisiones de 1990 . 


\section{VENTAJAS Y BENEFICIOS EN EL USO DE LAS ENERGIAS RENOVABLES}

Frente a la situación comentada en el punto anterior, la solución pasa por potenciar el papel de las fuentes renovables. Estas energías son los únicos recursos energéticos inagotables, no emiten contaminantes a la atmósfera y pueden ser aprovechados durante un tiempo indefinido. Las ventajas son tantas (aunque tampoco faltan, como veremos, los inconvenientes e impactos medioambientales) que se convierten necesariamente en la opción energética del futuro. Además cuentan con un brillante pasado, ya que hasta el siglo XIX cubrían la práctica totalidad de las necesidades energéticas. Sólo a partir de entonces empezaron a ser superadas por los combustibles fósiles, sobre todo el carbón y el petróleo.

Estas energías saben dar respuesta a la demanda social de reducción de contaminantes por su baja agresión ambiental en comparación con las energías tradicionales. La creciente participación de la electricidad en el consumo final de energía puede favorecer un aumento de las renovables debido a su mayoritaria especialización en la generación eléctrica. Su mayor problema es que su coste final es muy superior al correspondiente a las energías tradicionales. Habrá que descartar, en cualquier caso, todo criterio que sea exclusivamente economicista, ya que si se introducen criterios medioambientales las renovables resultan ampliamente competitivas (Jarabo, F. y otros, 1991).

En la tabla 4 se muestran, como ejemplo, los resultados obtenidos de una reciente investigación realizada en España, con un estudio comparativo de los impactos ocasionados por centrales de carbón, gas natural y eólicas para la producción eléctrica, indicando los valores de emisión de los tres principales compuestos contaminantes: $\mathrm{CO}_{2}, \mathrm{SO}_{2}$ y $\mathrm{NO}_{x}$.

Tabla 4. Emisiones con diferentes tecnologias de producción eléctrica en España ( $g / K W h)$

\begin{tabular}{cccc}
\hline Contaminante & Carbón & Gas natural & Eólica \\
\hline $\mathrm{CO}_{2}$ & 1.026 & 402 & 14,9 \\
$\mathrm{SO}_{2}$ & 1,2 & 0,2 & 0,1 \\
$\mathrm{NO}$ & 1,8 & 0,3 & 0,06 \\
\hline
\end{tabular}

Fuente: CIEMAT.

En estos datos están consideradas las fases de extracción de combustible, fabricación de materiales, transporte, generación eléctrica y depósito de residuos. Sólo de esta forma se estima de forma global el im- 
pacto ambiental de las actividades de generación eléctrica, incluidos el desmantelamiento de las instalaciones una vez finalizada su vida útil. En este último caso, las centrales térmicas convencionales y nucleares resultan especialmente inconvenientes debido a los problemas relacionados con la recuperación de suelos contaminados. En el caso nuclear, esto se ve agravado por el carácter radiactivo de los residuos y de las instalaciones que estuvieron en contacto directo o indirecto con el combustible y sus radiaciones.

El uso de las renovables está evitando anualmente la emisión de unos 345 millones de toneladas de dióxido de carbono en todo el mundo, según datos proporcionados por la Agencia Internacional de la Energía. Las reducciones también afectan al óxido de nitrógeno y al dióxido de azufre. En general, están en condiciones de modificar el actual panorama de emisiones de gases de efecto invernadero, tanto que se prevé, desde el Consejo Mundial de la Energía, reducir el $\mathrm{CO}_{2}$ en unos 9.000 millones de toneladas para el 2.020. En cuanto al dióxido de azufre y los óxidos de nitrógeno, las cifras oscilan en torno a los 13 y 22 millones de toneladas, respectivamente.

Además de la reducción de las emisiones a la atmósfera, las renovables presentan otros beneficios medioambientales. En primer lugar, la mejora en el abastecimiento de agua potable: mediante el uso de pequeñas máquinas eólicas se puede extraer el agua subterránea hasta la superficie para su consumo humano. En aquellas zonas donde hay sobreproducción de alimentos, la introducción de cultivos energéticos (que no requieren elementos químicos en su desarrollo) puede aliviar la excesiva contaminación de las aguas y mantener productivas, además, muchas regiones del planeta. Al mismo tiempo, pueden emplearse diversas tecnologías de energías renovables para la potabilización del agua en zonas costeras, especialmente en aquéllas de clima desértico.

En segundo lugar, el uso de las renovables a nivel local o en comunidades apartadas evita la construcción de sistemas necesarios para la transmisión y distribución de electricidad y, con ello, el impacto visual que dichos sisiemas ocasionan sobre el paisaje desde el punto de producción hasta el de consumo.

$Y$, en tercer lugar, la introducción de cultivos energéticos en tierras degradadas por prácticas agrícolas previas contribuye a mejorar las condiciones del suelo y lo previene de la posible erosión. Esto es especialmente importante en áreas marginales. Ahora bien, si estos cultivos entrañan la reducción de los espacios agrícolas tradicionales o detraen ciertos cultivos alimenticios para la producción de energía, es más recomendable 
aprovechar al máximo los residuos como fuente de energía y dedicar la mayor superficie posible a cultivos, sobre todo en los países del Tercer Mundo y con fuerte presión demográfica.

Los beneficios, por lo tanto, son evidentes y suponen un mayor respeto hacia el medio. Aparte de lo ya enunciado, cabe destacar también que no producen residuos de difícil eliminación, utilizan recursos que no se agotan a escala humana, los impactos medioambientales no tienen carácter permanente, ya que son reversibles y se dan más en la fase de construcción que en la de explotación, y generan una energía descentralizada allí donde existen recursos naturales suficientes. Como efectos positivos específicos cabe apuntar los siguientes:

a) Energía eólica. Se ha calculado que un parque de $10 \mathrm{MW}$ evitaría la emisión a la atmósfera de unas 22.500 toneladas anuales de $\mathrm{CO}_{2}$, principal gas de efecto invernadero y que se verterían utilizando las centrales térmicas convencionales. También hay que apuntar que no existen efectos negativos sobre el clima, la geomorfología o la hidrología, ni riesgo de vertidos accidentales, al igual que sucede con la energía solar.

b) Energía solar térmica. Al margen del campo puramente energético, la aplicación de esta energía en el sector turístico (hoteles y apartamentos) crea una oferta de calidad y de respeto al medio que puede ser especialmente valorado por el cliente. Como ocurre con la solar fotovoltaica, el aprovechamiento se caracteriza por la ausencia de ruidos o cualquier tipo de emisión a la atmóstera o el agua.

c) Biomasa. La reutilización de residuos orgánicos ayuda a resolver el problema ambienta! que genera su falta de tratamiento. La eliminación de los residuos agrícolas leñosos y herbáceos disminuye el riesgo de incendios ocasionados por quemas no controladas o la posible aparición de plagas. El aprovechamiento energético de los restos de algunas fábricas evita el vertido de materiales con fuerte carga contaminante, como en el caso de la industria oleícola. El uso de biocombustibles es muy interesante de cara a reducir las emisiones por circulación de vehículos, sobre todo en las áreas urbanas. Además, también hay que considerar que el bioetanol y el biometano son biodegradables y carecen prácticamente de azufre y cloro en su composición.

d) Hidráulica. Las presas que se construyen con fines energéticos regulan las corrientes fluviales y se reduce el riesgo de avenidas y estiajes prolongados, permitiendo su uso para el riego, el abastecimiento de poblaciones y los fines recreativos. 
Además de los beneficios medioambientales, hay otros de tipo socioeconómico igualmente importantes. Sin embargo, en el apoyo dado por parte de los gobiernos a las energías renovables siempre han pesado más los vinculados con el medio ambiente, considerándose todavía hoy los demás como beneficios adicionales y secundarios.

Estas fuentes energéticas gozan ya de un buen nivel tecnológico y de comercialización, sobre todo en lo relativo a la hidráulica, eólica, biomasa y solar térmica. Ello ha supuesto un gran incentivo para las industrias relacionadas con el sector y para el empleo, ya que se han abierto numerosas oportunidades al respecto. Estas tecnologías son intensivas en capital, mucho más que las propias de las energías no renovables. Las mejoras deben ir encaminadas al uso de nuevos materiales y a la reducción del precio de los componentes.

Esto es fundamental de cara a incrementar la competitividad industrial. La energía es un recurso básico para la actividad industrial, tanto para los procesos productivos como para la distribución de los productos. Su coste es clave, sobre todo allí donde su utilización es más intensiva, como la siderurgia, metalurgia, química, petroquímica, fabricación de cemento y materiales de construcción. La mejora tecnológica de las renovables como recurso energético repercute necesariamente sobre la industria de forma muy positiva y, con ello, sobre otros sectores económicos.

La industria de las energías renovables ha crecido de forma muy considerable en los últimos años en la mayor parte de los países desarrollados. Europa es un ejemplo, tal y como reconoce el llamado Libro Blanco de la Energía aprobado en 1997: en él se recogen las posibilidades de exportación de tecnología a países de Asia, África y América Latina con expectativas de crecimiento en el campo de las renovables. Para el año 2010, la Unión Europea ha calculado que las exportaciones del sector podrán alcanzar los 17.000 millones de euros.

Para el caso de España, se calcula que el sector de las energías renovables alcanza un nivel de negocio superior a los 600 millones de euros anuales, con unas 500 empresas en actividad, mayoritariamente de pequeño y mediano tamaño y localizadas sobre todo en Cataluña, País Vasco, Madrid, Andalucia y Valencia. El $50 \%$ de estas empresas tiene entre 1 y 25 trabajadores, el $17 \%$ entre 25 y 50 , el $12 \%$ entre 50 y 100 , el $16 \%$ entre 100 y 500 y sólo el $5 \%$ tiene más de 500 empleados. Es un entramado industrial, por tanto, con un alto potencial de creación de empleo.

Los recursos renovables aparecen concentrados a menudo en áreas rurales con escasas oportunidades de trabajo, altas tasas de desempleo y poblaciones en declive. El uso de estos recursos puede incentivar la eco- 
nomía local e incrementar de forma efectiva el número de trabajos. Las energías renovables se convierten en una especie de potencial endógeno que contribuye al crecimiento equilibrado de las regiones. No obstante, la materialización de estas ventajas necesita de un importante esfuerzo financiero y de promoción por parte de todas las administraciones.

La creación de empleo es uno de los efectos más positivos de la penetración de las fuentes de energía renovables. Numerosos estudios se han llevado a cabo en el ámbito europeo. En 1996, un estudio realizado para la Comisión Europea, y recogido en el ya citado Libro Blanco, apuntaba la creación de 500.000 empleos para el año 2010 en los quince paises, creados directamente por el sector de las renovables y por otros sectores que lo abastecen. En estas cifras aparecían ya descontadas las posibles pérdidas de empleo en otros sectores energéticos. En 1999, otro estudio (en el que han participado varias agencias europeas), dentro del programa ALTENER y titulado «The Impact of Renewables on Employment and Economic Growth», señala la importancia en cuanto a la nueva creación de puestos de trabajo, elevando la cifra a 660.812 para el año 2010 y a 900.547 para el 2020. En torno al $8 \%$ de los mismos correspondería a España.

En dicho estudio se comenta la trascendencia de las renovables en el desarrollo regional y la creación de empleo en áreas apartadas de la Unión Europea. Fue encargado en 1998 por la Comisión Europea, con el propósito de averiguar el impulso que dan estas energías al empleo y crecimiento económico. El año de partida es 1995 y se consideran tres periodos: 19952005,2010 y 2020 . Se recogen datos para los quince paises miembros.

El estudio prevé un incremento en la producción de energía renovable que resulta de multiplicar por 2,4 los valores iniciales de 1995, al pasar de 440 TWh en 1995 a 1.066 TWh en 2020. Esta evolución afecta positivamente a todos los Estados y a todos los sectores: en valores absolutos destaca la biomasa (combustión y digestión anaerobia) y en valores relativos la energía eólica y la solar fotovoltaica.

Como resultado del incremento anterior en la producción se prevé un claro aumento del empleo relacionado directa o indirectamente con el sector energético de las renovables. Hasta el 2005 se calcula un impacto sobre el empleo de 453.418 nuevos puestos de trabajo, cifra que se eleva a 660.812 en 2010 y 900.547 en 2020 . La mayoría del empleo se creará en las tecnologías vinculadas con la biomasa, y eso sin desplazar al empleo de la agricultura convencional. Otras tecnologías generarán unos niveles de empleo más modestos, como la eólica. La solar fotovoltaica y la minihidráulica perderán empleo los primeros años pero acabarán recuperándose hacia el 2020. Hay que tener en cuenta que, en general, las ener- 
gías renovables son más intensivas en mano de obra que las tecnologías energéticas tradicionales.

Tabla 5. Creación de empleo con renovables en la Unión Europea sobre 1995

\begin{tabular}{lrrr}
\hline \multicolumn{1}{c}{ Energia renovable } & \multicolumn{1}{c}{2005} & \multicolumn{1}{c}{2010} & \multicolumn{1}{c}{2020} \\
\hline Solar térmica & 4.590 & 7.390 & 14.311 \\
Solar fotovoltaica & 479 & -1.769 & 10.231 \\
Solar termoeléctrica & 593 & 649 & 621 \\
Eólica & 9.220 & 12.854 & 28.627 \\
Minihidráulica & -11.391 & -995 & 7.977 \\
Biocarburantes & 37.223 & 70.168 & 120.285 \\
Biogás & 15.640 & 27.582 & 37.271 \\
Biomasa de biocombustibles & 89.424 & 128.395 & 165.860 \\
Producción de & 307.641 & 416.538 & 515.364 \\
$\quad$ Total & 453.419 & 660.812 & 900.547 \\
\hline
\end{tabular}

Fuente: Comisión Europea (1999): Proyecto ALTENER.

Por países, los incrementos absolutos más importantes se registran en Alemania, Francia e Italia, mientras que Dinamarca, Grecia y Austria lo hacen en relación con el tamaño de su fuerza de trabajo. Los resultados para los quince Estados miembros quedan recogidos en la tabla 6 y también se toma como base el año 1995.

Tabla 6. Creación de empleo en los quince países miembros

\begin{tabular}{|c|c|c|c|}
\hline País & 2005 & 2010 & 2020 \\
\hline Bélgica & 4.040 & 4.605 & 6.936 \\
\hline Dinamarca & 58.758 & 64.546 & 73.539 \\
\hline Alemania & 81.282 & 134.618 & 183.759 \\
\hline Grecia & 17.311 & 46.385 & 83.470 \\
\hline España & 37.389 & 44.971 & 84.397 \\
\hline Francia & 87.018 & 126.832 & 135.164 \\
\hline Irlanda & 4.446 & 7.981 & 11.184 \\
\hline italia & 21.405 & 66.201 & 132.077 \\
\hline Luxemburgo & 353 & 353 & 353 \\
\hline Holanda & 13.306 & 5.901 & 8.464 \\
\hline Austria & 55.746 & 59.980 & 62.182 \\
\hline Portugal & 26.778 & 38.116 & 47.473 \\
\hline Finlandia & 20.695 & 26.071 & 30.592 \\
\hline Suecia & 15.437 & 19.098 & 22.583 \\
\hline Reino Unido & 9.453 & 15.155 & 18.373 \\
\hline Total & 453.419 & 660.812 & 900.547 \\
\hline
\end{tabular}

Fuente: Comisión Europea (1999): Proyecto ALTENER 
Estos datos confirman la idea de que las energías renovables presentan un impacto muy positivo sobre el mercado de trabajo y el crecimiento económico. La formación de nuevos profesionales está garantizada en los países desarrollados pero no en los del Tercer Mundo. El uso de estas energías es muy importante para los Estados subdesarrollados, ya que reduce la dependencia energética del exterior. Además, disponen en principio de un elevado potencial en energías renovables: la mayor parte se localizan en la zona intertropical, en donde la insolación es intensa y las precipitaciones abundantes. El uso de estos recursos podría convertirse en una opción descentralizada y más barata de electrificación rural y, por lo tanto, de modernización y desarrollo. La idea está ampliamente aceptada pero falta a menudo el capital necesario para realizar las inversiones.

\section{IMPACTOS ASOCIADOS A LAS ENERGIAS RENOVABLES}

Las energías renovables forman un amplio sistema de posibilidades energéticas. No se agotan, se renuevan y presentan todas las ventajas ambientales y socioeconómicas ya comentadas. Sin embargo, también existen ciertos impactos sobre el medio que es imprescindible comentar. Antes de hacerlo individualmente para cada recurso renovable, hay que advertir que todos son de ámbito local y con repercusiones muy concretas. Estos problemas permiten acciones para su control relativamente simples y, desde el punto de vista económico, no muy costosas. Por lo tanto, en absoluto comparables con los graves problemas a todas las escalas que plantean los combustibles fósiles.

En la fase de explotación, las energias renovables no emiten contaminantes a la atmósfera. Sus problemas son de tipo visual (aerogeneradores y plantas solares), acústico (aerogeneradores), oloroso (plantas de biogás) o de alteración de la ecología de los cursos fluviales (presas hidroeléctricas). También existen los de tipo social y económico: abandono de pueblos anegados por presas, emigración hacia otros lugares de residencia, desarraigo, pérdida de tierras de cultivo, etc. En cualquier caso, y a pesar de que algunos de estos impactos son inevitables, se pueden tomar algunas medidas correctoras que limiten la intensidad o agresividad de la acción.

Los impactos ambientales de las centrales hidroeléctricas son, en general, reducidos, aunque pueden adquirir mayor relevancia en el caso de centrales de gran tamaño o dependiendo de su situación geográfica y su entorno físico, biológico y climático. Las alteraciones más acusadas se dan en la fase de construcción: transporte de materiales, ruidos, polvo, incremento de la materia en suspensión en las aguas, modificación de la flora y 
fauna de la zona, etc. Sin embargo, el periodo de construcción no es excesivamente largo y la mayor parte de los impactos se acaban eliminando.

En la fase de explotación de la central, la calidad del agua se puede ver alterada: por deficiencia de oxígeno en el vaso y por acumulación de sedimentos, ya que las características de erosión y deposición de un río se ven afectadas. Las consecuencias pueden ser importantes en llanuras aluviales y deltas aguas abajo, en donde la agricultura y los ecosistemas naturales dependen en gran medida de los nutrientes en suspensión en el agua. La presencia de presas impide, además, la migración de las especies a lo largo del río. Si su tamaño es grande, hay también repercusiones en el clima local, aumentando la humedad. Se pueden llegar a formar, incluso, neblinas como las que se dan en los lagos naturales.

Todos estos problemas ambientales se ven compensados por algunos beneficios de indudable interés, como el abastecimiento de agua para la población, la industria y la agricultura, el control de las corrientes, el aumento del empleo o la reducción en la importación de combustibles. Cuando los impactos resulten inevitables habrá que tomar las medidas necesarias para reducir, cambiar o compensar dichos impactos, así como los de tipo residual que pudieran surgir.

La energía solar térmica no afecta ni a la calidad del aire, ni a los suelos ni a las aguas y no provoca ruidos. El principal impacto es el visual sobre el paisaje que se deriva de las instalaciones necesarias para la explotación de este recurso renovable. Los sistemas solares térmicos deben de integrarse de forma lo más respetuosa posible en el medio. Cuando se instalan en la ciudad, son los edificios los que exigen la mejor adaptación. La flora y la fauna sólo se ven afectadas cuando las centrales solares térmicas ocupan grandes extensiones. En cualquier caso hay que tener en cuenta que las tecnologías aplicadas a las solares térmicas son de las más eficientes en la relación superficie/producción eléctrica dentro del campo de las renovables, con un dato aproximado de 0,25 hectáreas/GWh/año.

Las cargas ambientales derivadas de la energía solar fotovoltaica se dan en las operaciones de extracción de materias primas. Como la mayoría de las células fotovoltaicas son de silicio (obtenido a partir de la arena, muy abundante), los problemas tampoco son graves. Uno de los principales efectos sobre el ecosistema es la construcción de grandes plantas fotovoltaicas, que logran remover grandes cantidades de tierra y alteran la flora y fauna del lugar, además de los efectos visuales en la zona. En la fase de utilización energética de las instalaciones, los impactos son prácticamente despreciables sobre el medio físico, aunque es necesario tener 
cuidado con la correcta gestión de los módulos fotovoltaicos una vez agotada su vida útil, intentando reciclar y reutilizar lo máximo posible.

Los impactos asociados a los cultivos energéticos (para la producción de biocombustibles como el etanol) varian en función del tipo de cultivo y de las labores realizadas en las plantaciones energéticas. El uso de productos agroquímicos es más bajo que con los cultivos normales: los pesticidas, insecticidas y fungicidas no siempre se utilizan y cuando se hace, siempre en menores cantidades. La relación superficie/producción eléctrica hace a estos cultivos energéticos los sistemas más extensivos en el ámbito de los recursos renovables: 60 hectáreas/GWh/año. De ello se derivan enormes extensiones de monocultivos energéticos cuyo efecto visual es innegable. Se puede reducir adaptándolos a la topografía del lugar y usando diferentes plantas. En resumen, los cultivos energéticos plantean pocos impactos ambientales y siempre son de menor escala que con los cultivos tradicionales. La puesta en explotación de tierras marginales, evitando su erosión es, por si sola, una ventaja considerable que justifica su extensión, siempre y cuando no se den circunstancias de escasez de alimentos.

La digestión anaerobia (fermentación de residuos vegetales en ausencia de oxígeno) crea un gas (biogás o gas biológico) que puede transformarse en energía calorífica mediante combustión. Este proceso ayuda a resolver el problema de tratamiento de los residuos orgánicos contaminantes y su transformación en productos libres de microorganismos patógenos. Los efectos negativos sobre el medio ambiente podrian derivarse de la percolación de materia orgánica o metales pesados hacia aguas subterráneas o superficiales procedentes de los lodos resultantes del proceso de digestión anaerobia. Las instalaciones suelen ocupar amplias superficies, con efectos evidentes sobre el paisaje de tipo visual; pero también por los inevitables malos olores de una planta de estas características, por lo que se hace imprescindible un estudio detallado de ubicación.

Los sistemas geotérmicos también provocan impactos sobre el medio ambiente. Dependiendo de su localización, las instalaciones pueden alterar los ecosistemas locales por ruido, humos y erosión del terreno. El ruido se produce durante las fases de exploración, construcción y producción, en niveles que a veces alcanzan el umbral de los $120 \mathrm{dBa}$. Las emisiones gaseosas a la atmósfera también están presentes en forma de gases no condensables (cuando el sistema no reutiliza los fluidos de trabajo en un circuito cerrado): dióxido de carbono y sulfuro de hidrógeno. Estas emisiones pueden ser reducidas mediante el uso de diferentes tecnologías que las moderan.

También la geotérmica puede contaminar las aguas superficiales a través de los distintos fluidos e incidir sobre la vegetación y la fauna acuáticas. Estos 
impactos son casi inexistentes en los sistemas más avanzados porque se vuelven a utilizar los fluidos reinyectándolos de nuevo en las rocas del campo geotérmico. Esta operación debe de evitar cualquier tipo de fallo para impedir el impacto sobre los acuíferos subterráneos. Además, como las plantas geotérmicas se localizan en regiones tectónicamente activas, la reinyección puede incrementar la frecuencia de los pequeños terremotos. Los efectos se reducen controlando al máximo la reinyección de los fluidos y utilizando materiales resistentes a la sismicidad en las instalaciones del campo.

Las plantas geotérmicas son, en general, poco llamativas. Sin embargo, su impacto visual puede ser significativo porque se suelen situar en áreas de excepcional belleza natural y de fuerte atracción turística (géiseres, lagunas calientes...).

Las molestias provocadas por las energías renovables tienen efectos localizados y reversibles y a menudo se superan a través de diversas soluciones técnicas sin influir en la fuente de energía. No representan ningún peligro serio para el medio ambiente, muy al contrario de lo que sucede con las formas tradicionales, cuyos impactos son de larga duración y costosos en su eliminación. Se presentan en el cuadro adjunto los principales impactos de carácter medioambiental producidos durante la fase de explotación o generación eléctrica de los distintos recursos renovables tratados hasta aquí; para completar el panorama se incluye anticipadamente a la energía eólica, objeto de estudio en el apartado siguiente.

Tabla 7. Impactos ambientales durante la fase de explotación

\begin{tabular}{lll}
\hline \multicolumn{1}{c}{ Energía } & \multicolumn{1}{c}{ Impactos principales } & Nivel de impacto \\
\hline Hidráulica & Alteración de los cursos fluviales & Alto \\
& Modificación del clima local & Medio \\
& Erosión del suelo & Alto \\
Solar térmica & Impacto visual & Medio \\
& Alteración de los ecosistemas & Bajo \\
Solar fotovoltaica & Impacto visual & Medio \\
& Alteración de los ecosistemas & Bajo \\
Cultivos energéticos & Contaminación del suelo y agua & Bajo \\
& Erosión del suelo & Bajo \\
Digestión anaerobia & Percolación de materia orgánica & Medio-alto \\
& Malos olores & Alto \\
& Impacto visual & Medio \\
Geotérmica & Emisiones gaseosas & Bajo-medio \\
& Ruido & Medio \\
Eólica & Degradación de paisajes naturales & Alto \\
& Ruido & Medio-alto \\
& Impacto visual & Alto \\
& Alteración de los ecosistemas & Bajo \\
\hline
\end{tabular}

Fuente: elaboración propia. 


\section{EL EJEMPLO DE LA ENERGÍA EÓLICA}

La generación de electricidad a partir del viento presenta grandes ventajas desde el punto de vista medioambiental, ya que no produce gases tóxicos, no contribuye al efecto invernadero ni a la lluvia ácida y no origina residuos radiactivos. Cada kilovatio hora de electricidad producida con un aerogenerador, en sustitución del carbón, evita la emisión a la atmósfera de un kilogramo de $\mathrm{CO}_{2}$. Estas ventajas están en la base del espectacular desarrollo tecnológico experimentado en los últimos años por la energía eólica.

Cualquier proyecto eólico requiere, para su aprobación, un estudio de impacto medioambiental que se ha de presentar a la consejería con competencias en materia de medio ambiente de la respectiva Comunidad Autónoma. El impacto de esta energia depende de tres factores principales: del carácter del proyecto en sí mismo; de la fragilidad ecológica del territorio en el que se vaya a localizar el proyecto; y de la calidad ecológica del lugar de ubicación del parque eólico. Los proyectos en sí generan poco impacto, por lo que hay que centrarse fundamentalmente en la calidad y fragilidad ecológica del territorio, atendiendo tanto a la flora como a la avifauna, el impacto visual y el ruido.

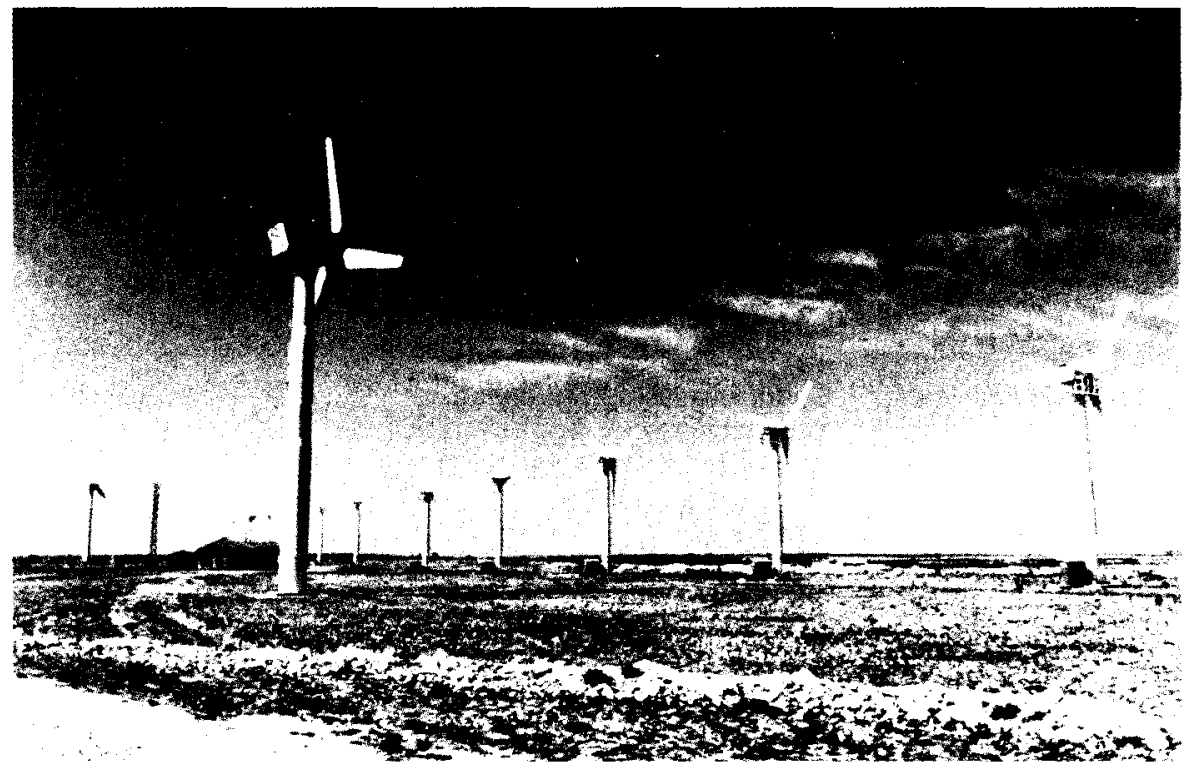

Fig. 2. Parque eólico "La Muela" (Zaragoza). 
Los efectos de la energía eólica sobre la flora son de escasa influencia y se reducen sólo a la fase de construcción del parque (movimiento de tierras, preparación de accesos, cimentaciones para los aerogeneradores, edificios de control, etc.) Los efectos son más importantes sobre la avifauna, sobre todo en el caso de aves planeadoras. El riesgo aumenta a medida que son más altos los molinos y sus palas giran a mayor velocidad. En cualquier caso, se ha comprobado que existe menor peligro de muerte para las aves por colisión con un aerogenerador que con un tendido eléctrico (CIEMAT, 1997).

Para las aves migratorias diurnas, el peligro de colisión es insignificante, ya que con buenas condiciones meteorológicas divisan fácilmente el obstáculo y modifican su recorrido a gran distancia del parque eólico. Algo muy distinto sucede con las aves migratorias nocturnas, en donde aumenta considerablemente el peligro de colisión. Esto se reduce evitando la instalación de molinos en las áreas que coincidan con las rutas de migración de las aves o con los espacios de reproducción de especies protegidas. Es la mejor medida para minimizar los efectos adversos.

Para la opinión pública, el mayor inconveniente que presenta el uso de la energia eólica es el del impacto visual. Aunque sea el menos cuantificable, y el más subjetivo, no puede obviarse este problema. Este impacto depende tanto de la capacidad del observador para registrar las impresiones visuales como del paisaje (topografía, vegetación, edificios, etc.) y de las características del propio parque (tamaño, altura de los molinos, material y color).

Los efectos visuales, después de numerosos estudios, tienen una serie de límites físicos que se corresponden con cuatro zonas distintas, según un estudio del CIEMAT (1997): la zona I tiene el límite marcado por el radio de las palas; en la zona II, el límite es tres veces la altura del aerogenerador y a esta distancia el observador aumenta el control del objeto alto; el límite de la zona III se ha estimado en diez veces la altura y en esta zona la turbina domina un sector del campo de visión; en la zona IV, la turbina es percibida como un elemento más del paisaje lejano y cuyos límites vienen determinados por las condiciones de visibilidad, el tiempo, la topografía, la vegetación y la presencia de edificios. La longitud teórica de visibilidad en el caso de un paisaje llano y abierto es de 10 kilómetros por cada 25 metros de turbina. Los molinos son estructuras verticales que destacan con más facilidad en paisajes horizontales. Además, las palas en movimiento atraen la atención del observador y forman elementos dominantes en el paisaje. 
El ruido que produce un aerogenerador es similar al de cualquier otro equipamiento industrial de la misma potencia. El efecto nocivo se debe a que mientras los equipos convencionales se encuentran encerrados en edificios, los molinos trabajan al aire libre. El ruido se debe a factores de tipo mecánico (multiplicador, generador, ventilador del generador) y de tipo aerodinámico (velocidad del giro del rotor, material de fabricación de las palas, superficie aerodinámica de las mismas). En todo caso, las molestias van a depender de la distancia del parque eólico respecto a la población residente en las localidades de la zona, aumentando en el caso de casas aisladas próximas a las instalaciones.

\section{LAS ENERGIAS RENOVABLES: POR UN NUEVO MODELO ENERGÉTICO}

El actual modelo energético, basado fundamentalmente en el consumo de energías acumuladas a lo largo de diferentes eras geológicas, profundiza las desigualdades entre las sociedades y agrava los ya considerables problemas ambientales. Hay que potenciar las energías renovables (también llamadas "energías flujo"), que se renuevan constantemente y son inagotables.

Las energías renovables suponen la opción energética del futuro. Además cuentan con un brillante pasado en el que hasta bien entrado el siglo XIX cubrían la práctica totalidad de las necesidades energéticas de la sociedad. Sólo a partir de entonces fueron superadas por el carbón, el petróleo y, más tarde, el gas natural.

Las ventajas ambientales son evidentes y han quedado suficientemente comentadas a lo largo del artículo. Sin embargo, también es necesario destacar una serie de características para el conjunto de las energías renovables que limitan en mayor o menor medida las posibilidades de utilización. En primer lugar, aunque estas fuentes son universales, ya que aparecen en todos los puntos del planeta, afectan a las diferentes regiones del globo de forma muy desigual. En segundo lugar destaca la discontinuidad en el tiempo, como ocurre con la eólica y solar, por lo que no se puede captar la energía más que de forma secuencial y las interrupciones en la alimentación son inevitables y frecuentes. Por último, estas fuentes energéticas son con frecuencia aleatorias, ya que son tributarias en la mayor parte de los casos de las condiciones climáticas, que el hombre no sabe todavía prever de manera precisa. 
El nivel actual de utilización de las energías renovables es muy desigual según las zonas y parece vincularse con las sociedades más industrializadas. Las inversiones son todavía costosas y la tecnología cada vez más experimentada, lo que explica que las energías renovables no se hayan desarrollado extensamente en los países del Tercer Mundo. Por el contrario, los países ricos llevan apostando por estos recursos desde hace ya algún tiempo, con programas de ámbito nacional para el fomento de un sistema más equilibrado de consumo energético en el que las renovables jueguen cada vez un papel más importante.

En diciembre de 1999 fue aprobado en España el llamado Plan de Fomento de las Energías Renovables para el periodo 1999-2010. El Plan establece unos ambiciosos objetivos de desarrollo dirigidos a que las energías renovables cubran al menos el $12 \%$ de la demanda total de energía en el 2010 (actualmente aportan en torno al 6,5\% del balance energético nacional). Ese objetivo coincide con el global para la Unión Europea fijado en el Libro Blanco de las Energías Renovables.

El objetivo del Plan es ambicioso por dos razones: en primer lugar, porque duplicar la participación de estas fuentes (teniendo en cuenta el contexto general de crecimiento de la demanda energética) supone multiplicar por más de dos la cantidad que deben aportar las renovables. Y en segundo lugar, porque el grueso de la contribución actual de estas energías proviene de la hidráulica y biomasa, la primera con perspectivas limitadas de desarrollo (sobre todo la gran hidráulica) y la segunda deberá de incorporar nuevas formas de uso y obtención de recursos para alcanzar las cifras inicialmente asignadas.

El mayor crecimiento previsto es el de la biomasa, con un objetivo de aumentar en 6 millones de tep (toneladas equivalentes de petróleo). La energía eólica es la segunda en importancia por su aportación al consumo de energía primaria en el año 2010. Entre los objetivos principales también se encuentra el impulso de la solar térmica y fotovoltaica. Para la energía solar térmica de baja temperatura, de la que en la actualidad hay instalados cerca de 341.000 metros cuadrados de paneles solares, se prevé la instalación a lo largo del periodo de vigencia del Plan de 4.500.000 metros cuadrados.

Los biocarburantes constituyen un área de nuevo desarrollo y su contribución será de $500 \mathrm{ktep}$ en el 2010. El biogás experimentará al mismo tiempo un crecimiento sustancial, al igual que el aprovechamiento energético de los residuos sólidos. La minihidráulica aumentará cerca de un $50 \%$, mientras que la hidráulica de potencia superior a $10 \mathrm{MW}$ apenas registra incremento. 
Tabla 8. Situación actual y objetivos del Plan de Fomento de las Energías Renovables (producción energética en ktep)

\begin{tabular}{lcc}
\hline \multicolumn{1}{c}{ Área } & Situación actual & Objetivos para 2010 \\
\hline Minihidráulica & 402 & 594 \\
Hidráulica (10-50 MW) & 482 & 542 \\
Hidráulica & 2.135 & 2.135 \\
Eólica & 172 & 1.852 \\
Biomasa & 3.645 & 9.645 \\
Biogás & - & 150 \\
Solar fotovoltaica & 1,3 & 19 \\
Solar termoeléctrica & - & 180 \\
Solar térmica de baja termperatura & 26 & 336 \\
Residuos sólidos & 247 & 683 \\
Geotermia & 3 & 3 \\
Biocarburantes & - & 500 \\
\multicolumn{1}{c}{ Total } & 7.113 & 16.639 \\
\hline
\end{tabular}

Fuente: MINER (1999): Plan de Fomento de las Energias Renovables.

La importancia medioambiental de este Plan radica en su contribución a la limitación de emisiones de $\mathrm{CO}_{2}$ a la atmósfera, tanto en lo que se refiere a la fase de producción de electricidad como a la utilización de energías para usos térmicos. El incremento previsto de las energías renovables supone evitar en el año 2010 la emisión de 41,5 millones de toneladas de $\mathrm{CO}_{2}$, de los que 36,5 corresponden a la generación de electricidad y 5 a los usos térmicos. La comparación de estas cifras con las emisiones registradas en 1990 (año base de referencia en el Protocolo de Kioto) destaca el potencial del Plan de Fomento frente a las fuentes fósiles desde el punto de vista medioambiental: $20 \%$ menos de emisiones de $\mathrm{CO}_{2}$ de origen energético en el 2010 respecto a 1990 y $18 \%$ menos de emisiones totales de $\mathrm{CO}_{2}$ para el mismo periodo.

Las ventajas de tipo medioambiental de las energías renovables contempladas en el Plan convierten a estos recursos energéticos en un elemento clave para el cumplimiento de los compromisos derivados del Protocolo de Kioto en el periodo 2008-2012 y de otros objetivos propuestos a corto y medio plazo.

\section{BIBLIOGRAFIA}

Azcárate Luxan, B. y Mingorance Jiménez, A. (2002): Energias e impacto ambiental, Madrid, Equipo Sirius, col. "Milenium".

BlanCo, A. (1996): La energia y la degradación del suelo, Madrid, Instituto Español de la Energía. CIEMAT (1997): Principios de conversión de la energía eólica, Madrid. 
COMISIÓN DE LAS COMUNIDADES EUROPEAS (1997): Energia para el futuro: fuentes de energía renovables, Libro Blanco para una Estrategia y un Plan de Acción Comunitarios.

Domínguez GARRIDO, V. (1994): Energias renovables y medio ambiente, Valladolid, Universidad de Valladolid.

EUROPEAN FORUM FOR RENEWABLE ENERGY SOURCES (1999): Impact of Renewables on Employment and Economic Growth, Luxemburgo.

FERNÁNDEZ, J. (1997): "La biomasa como alternativa energética", Tecnoambiente, 71, págs. 45 51.

FORO NUCLEAR (1999): Energía 1999, Madrid.

IDAE (1989): Guia metodológica de evaluación de impacto ambiental en pequeñas centrales hidroeléctricas, Madrid.

- (1999): Jornadas sobre energía eólica, Santiago de Compostela.

JaRABo Friedrich, F. (1991): El libro de las energias renovables, Madrid, Sociedad Anónima de Publicaciones Técnicas.

LUCENA BONNY, A. (1998): Energias alternativas y tradicionales. Sus problemas ambientales, Madrid, Talasa Ediciones.

Menéndez Perez, E. (1997): Las energias renovables. Un enfoque político-ecológico, Madrid, Los Libros de la Catarata.

MINER (1999): Plan de Fomento de las Energias Renovables, Madrid, Instituto para la Diversificación y Ahorro de la Energía.

Ortiz Torres, L. y Miguez TABares, J. L. (1995) (Coord.): Energias renovables y medio ambiente, Vigo, Universidad de Vigo.

PARdo ABAD, C. J. (1993): Las fuentes de energía, Madrid, Sintesis, Col. «Espacios y Sociedades", n. 22.

- (2001): "Situación actual del sector energético en España", Espacio, Tiempo y Forma "Geografía", 13, págs. 59-83.

RATTO, C. F. y SOLARI, G. (1998): Wind energy and landscape, Rotterdam, Balkema.

Ruiz Girela, J. (1997): “La energia hidráulica y el medio ambiente», Tecnoambiente, 71, págs. 5-12

SAYIGH, A. M. (1990): Energy and the environment, Oxford, Pergamon Press.

STERRETT, F.S. (1995): Alternative fuels and the environment, Florida, CRC Press.

Vazouez, M. y Morá, J. (1994): Energías limpias en progreso, Santiago de Compostela, Asociación Española de Energia Solar.

WAGNER, S. y otros (1996): Wind turbine noise, Berlín, Springer. 